\title{
Tracheal agenesis: Esophageal airway support with a 3-dimensional-printed bioresorbable splint
}

\author{
Anthony Y. Tsai, MD, ${ }^{\mathrm{a}}$ Morgan K. Moroi, MD, ${ }^{\mathrm{b}}$ Andrea S. Les, PhD,${ }^{\mathrm{c}}$ Scott J. Hollister, PhD, ${ }^{\mathrm{d}}$ \\ Glenn E. Green, MD, ${ }^{\mathrm{c}}$ Robert E. Cilley, MD, ${ }^{\mathrm{a}}$ Peter Dillon, MD, ${ }^{\mathrm{a}}$ and Joseph B. Clark, MD, ${ }^{\mathrm{e}}$ Hershey, Pa; \\ New York, NY; Ann Arbor, Mich; and Atlanta, Ga
}

\footnotetext{
From the Divisions of ${ }^{a}$ Pediatric Surgery and ${ }^{\mathrm{e}}$ Pediatric Cardiac Surgery, Penn State Health Children's Hospital, Hershey, Pa; ${ }^{b}$ Division of Cardiac, Thoracic, and Vascular Surgery, Department of Surgery, New York-Presbyterian Hospital/Columbia University Medical Center, New York, NY; ${ }^{c}$ Division of Otolaryngology-Head and Neck Surgery, University of Michigan, Ann Arbor, Mich; and ${ }^{\mathrm{d}}$ Wallace A. Coulter Department of Biomedical Engineering, Georgia Institute of Technology, Atlanta, Ga.

S.J.H. and G.E.G. were supported in part by National Institutes of Health/Eunice Kennedy Shriver National Institute of Child Health and Human Development R01 086201.

Disclosures: G.E.G. and S.J.H. are coinventors on an airway splint patent assigned to the Regents of the University of Michigan. This patent has been licensed by the University of Michigan to Materialise NV, Leuven, Belgium. All other authors reported no conflicts of interest.

The Journal policy requires editors and reviewers to disclose conflicts of interest and to decline handling or reviewing manuscripts for which they may have a conflict of interest. The editors and reviewers of this article have no conflicts of interest.

Received for publication Feb 19, 2021; accepted for publication Aug 26, 2021; available ahead of print Sept 3, 2021.

Address for reprints: Anthony Y. Tsai, MD, Division of Pediatric Surgery, Department of Surgery, Penn State Health Milton S. Hershey Medical Center, 500 University Dr, PO Box 850, Hershey, PA 17033 (E-mail: atsai1@ pennstatehealth.psu.edu).

JTCVS Techniques 2021;10:563-8

2666-2507

Copyright $(2021$ The Author(s). Published by Elsevier Inc. on behalf of The American Association for Thoracic Surgery. This is an open access article under the CC BY-NC-ND license (http://creativecommons.org/licenses/bync-nd/4.0/).

https://doi.org/10.1016/j.xjtc.2021.08.037
}

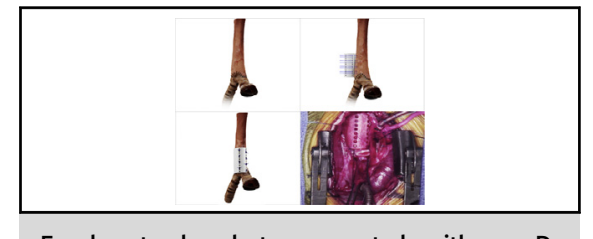

Esophagotracheoplasty supported with a 3Dprinted bioresorbable external splint.

CENTRAL MESSAGE

An esophageal airway support with a 3D-printed bioresorbable external splint can offer the opportunity for long-term survival for the typically lethal tracheal agenesis.

See Commentaries on pages 569 and 571.
Video clip is available online.

Tracheal agenesis (TA) is a rare and typically fatal congenital malformation that occurs in less than 1 in 50,000 live births. ${ }^{1,2}$ Approximately 150 cases have been reported, with near-universal lethality. ${ }^{3}$ TA is commonly classified into anatomical variations defined by Floyd (Figure E1). ${ }^{3}$ Newborns present with cyanosis, respiratory distress, and aphonia. Survival requires the presence of a tracheoesophageal fistula. Few successful airway reconstruction approaches have been described, with approximately 10 survivors. Survival beyond 1 year has been associated with externally supported esophageal airway reconstruction. ${ }^{1-4}$ Three-dimensional (3D) printing is an emerging modality for crafting implants for a variety of surgical populations. ${ }^{5}$ We report extended survival in a patient with TA through esophageal airway reconstruction with an externally supported 3D-printed bioresorbable splint.

\section{CLINICAL SUMMARY}

A term, 3.6-kg girl was born to a 35-year-old mother with limited prenatal care. The newborn showed respiratory distress and cardiovascular depression requiring chest compressions. Intubation was difficult but ultimately achieved. The baby stabilized and was supported with mechanical ventilation. With initiation of tube feeds, breast milk was visualized in the endotracheal tube. The patient was transferred to our institution on day of life (DOL) 2 for management of suspected tracheoesophageal fistula.

An esophagram showed an intact esophageal tract with contrast entering the airway, consistent with tracheoesophageal fistula. Additional studies demonstrated a complete atrioventricular canal defect, a right choroid plexus cyst, and hemivertebrae. Progressive abdominal distention compromised ventilation. Given confirmation of the esophagus-airway connection, and likely gastric outlet obstruction, the patient was taken to the operating room for pan-endoscopy and gastric decompression (DOL 3).

Flexible nasolaryngoscopy revealed a laryngeal cleft. Rigid bronchoscopy demonstrated a tracheoesophageal orifice in the mid-esophagus communicating to confluent mainstem bronchi. TA was diagnosed and a comprehensive 
consultation was conducted with the parents. The anticipated dismal prognosis was illustrated, and the option of comfort care was offered. A possible surgical plan and associated risks and benefits were also described. Ultimately, the parents expressed their strong desire to pursue potential lifesaving measures. After multidisciplinary discussion, consensus was reached to embark upon attempted rescue.

\section{Step 1 (DOL 3): Lower Esophageal Division and Gastrostomy}

To interrupt positive-pressure ventilation of the stomach, the lower esophagus was separated from the upper esophageal airway. Via laparotomy, the esophageal hiatus was opened, a paraesophageal dissection performed, the esophagus divided below the fistula with an endoscopic stapler, and the distal esophageal remnant was brought into the abdomen. The stomach was decompressed with gastrostomy tube placement. Duodenal obstruction was appreciated but treatment deferred.

Computed tomography (CT) imaging later confirmed (DOL 6) Floyd type I tracheal agenesis with a 2- to 4-mm diameter fistula connecting the mid-esophagus to a short tracheal segment. Duodenal obstruction and malrotation were addressed (DOL 8) with duodenojejunostomy and a Ladd procedure. Mucus plugging of the fistula occasionally necessitated bedside bronchoscopic clearance of secretions.

\section{Step 2 (DOL 15): Upper Esophageal Division With Airway Esophagostomy and Spit Fistula}

To provide a secure airway and divert oral secretions, the patient underwent neck dissection and division of the esophagus. The upper esophagus was diverted to a right cervical esophagostomy ("spit fistula"), and the middle esophagus externalized as a stoma above the sternal notch (airway esophagostomy, or "pseudo-tracheostomy"). The stoma was intubated with a 4.5-Fr, 44-mm extended tracheostomy tube.

The patient remained sedated and ventilated with positive pressure to minimize airway collapse. Gastrostomy feeds were initiated. Bedside bronchoscopy was intermittently performed for maintenance clearance of mucus.

A multidisciplinary team discussed the options for airway reconstruction. After evaluation of the reported experience and existing technologies, the decision was made to externally support the esophageal airway with a 3D-printed splint composed of slowly bioresorbable polycaprolactone (PCL). Similar splints had been successfully used to manage tracheobronchomalacia.

Permission for use of the device was obtained from the US Food and Drug Administration under Expanded Access, Penn State University institutional review board (Protocol No. 2018-002SU, March 28, 2018), and the Pennsylvania Department of Health. Using CT data imported into computer-aided design software, a virtual anatomic model was created and used for custom design of the airway device. Splints were 3D-printed using from $96 \%$ polycaprolactone and 4\% hydroxyapatite and sterilized with ethylene oxide according to established processes (Nelson Laboratories, Salt Lake City, Utah). Additional benchtop fit-testing was performed with life-size 3D-printed splints and airway models.

\section{Step 3 (DOL 143): Esophagotracheoplasty and Splint Placement}

After we obtained informed consent from the parents of the patient including the use of the 3D-printed splint under expanded access and manuscript publication related to the case, definitive airway reconstruction was performed through a median sternotomy with cardiopulmonary bypass support. Repair of the complete atrioventricular canal defect was accomplished first using a single-patch technique with cleft closure; epicardial echocardiography showed no residual shunts and trace-to-mild atrioventricular valve regurgitation. Airway reconstruction followed (Figure 1, Video 1). The esophagus, fistula, and mainstem bronchi were mobilized with preservation of the vagus nerves and vascular supply. A $15-\mathrm{mm}$ inner-diameter, 41-mm long PCL splint was selected and trimmed. The tracheoesophageal fistula was sharply resected (Figure 2, A). Incisions were carried from the lateral trachea onto each bronchus. The esophagus was divided and the distal remnant removed. An esophagotracheostomy anastomosis was fashioned using interrupted sutures (6-0 polydioxanone). Subsequently, partial-thickness mattress sutures (6-0 polypropylene) were placed in 5 longitudinal rows along the anterior and lateral aspects of the esophagus. The sutures were directed through the graft interstices, the splint parachuted down onto the airway, and the sutures tied. Bronchoscopy showed a large internal lumen without collapse during provocative suctioning. Cardiopulmonary bypass was concluded, the sternum closed, and the infant recovered to the intensive care unit.

Bronchoscopy for pulmonary toilet was performed daily during the first week and then less frequently; even in the absence of positive pressure, airway collapse was not observed (Figure E2). CT of the chest at 8.6 months showed a well-expanded esophageal airway without stenosis (Figure 2, B). At 11 months, the airway esophagostomy stoma was revised to address minor breakdown and the cervical esophagostomy dilated. The patient progressed and was discharged home at 13 months on a home ventilator program. CT of the chest at 23 months continued to demonstrate favorable airway anatomy (Figure 2, C); subsequent axial imaging has not been indicated. The patient eventually liberated from continuous mechanical ventilation at 2.4 years, spontaneously breathing on her own in the daytime with a heat-moisture exchanger and tracheostomy tube (Bivona 5-mm tracheostomy tube, 60-mm length; 


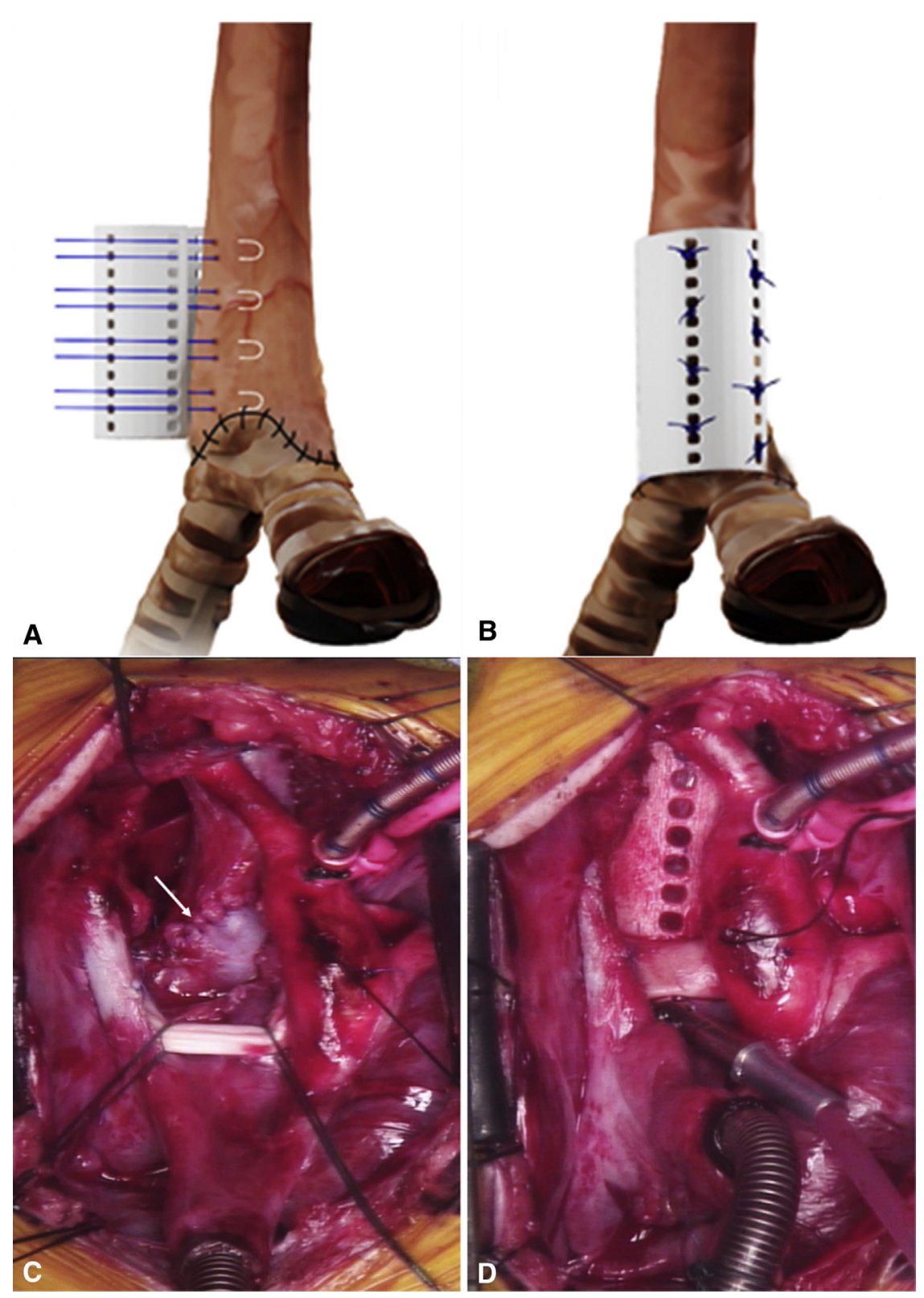

FIGURE 1. Repair of tracheal agenesis with esophagotracheoplasty and placement of a 3-dimensional-printed bioresorbable external splint. A, Following tracheoesophageal fistula resection and anastomosis of the esophagus to the trachea, rows of partial-thickness sutures are placed in the esophageal airway and brought through the splint interstices. B, The splint is parachuted onto the esophageal airway and sutures tied. C, Completed anastomosis of the esophagus to the trachea showing anterior sutures (arrow). D, Splint positioned around esophageal airway before suture placement.

Smiths Medical, Minneapolis, Minn), and resting overnight on ventilator support for safety.

\section{Step 4 (Age 2.6 Years): Restoration of Gastrointestinal Continuity}

Gastrointestinal continuity was re-established using a colon interposition graft to connect the cervical esophagus to the stomach. Working through the neck, right chest, and abdomen, the cervical esophagus and right colon were extensively mobilized and an intrathoracic anastomosis performed with interrupted sutures (4-0 polydioxanone). A pyloroplasty was added to augment gastric emptying. An upper gastrointestinal contrast study 6 weeks later showed an intact colon interposition graft without leak or obstruction (Figure E3).

At latest follow-up (age 3.4 years), the patient is alive and well and remains off ventilator support during the day. With speech therapy, she is progressing toward oral consumption 


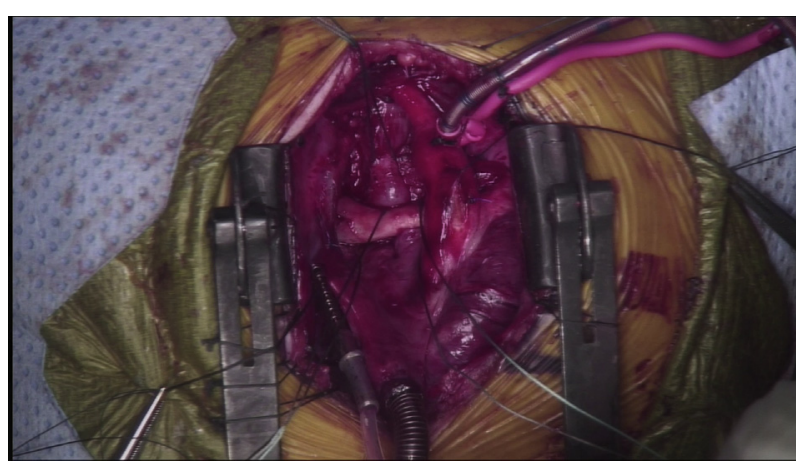

VIDEO 1. Operative video of the esophagotracheoplasty and splint placement with narration. Second half of the video demonstrates bronchoscopic videos of the reconstructed airway taken at 1 month and 18 months postoperatively, showing patency of the airway. Video available at: https://www. jtcvs.org/article/S2666-2507(21)00596-4/fulltext.

and is taking soft pureed foods by mouth. Aphonic, she uses basic sign language to communicate. Although developmentally delayed, she is crawling and cruising.

\section{DISCUSSION}

TA is a rare congenital anomaly with overwhelming early mortality and few precedent cases of successful management. Treatment requires creation of a substitute esophageal airway, sometimes aided by external or internal support. Ringed polytetrafluoroethylene (PTFE) grafts have been used as external support of the esophageal airway. ${ }^{1,4}$ Others have reported the use of rigid internal stents. ${ }^{6}$ While internal stents may provide satisfactory palliation, potential complications include obstruction, migration, and airway erosion. ${ }^{5}$ Fixed-size external implants similarly restrict airway growth and may require repeat surgery for upsizing. The use of synthetic materials for external stents has prompted concern for foreign body reaction, infection, and erosion. ${ }^{5}$

Bioresorbable materials such as PCL have shown recent success as airway splints and may offer potential benefits. ${ }^{5}$ The ability to use $3 \mathrm{D}$ printing allows for customized designs tailored to individual patient-specific needs. The present PCL splint is expected to slowly resorb over approximately 3 to 4 years, possibly providing both advantages of early airway support and the opportunity for future airway growth. Following resorption, if needed, a larger splint could be placed without the concomitant requirement for residual device explantation. Compared with ringed PTFE splints, which have a ribbed exterior and have been associated with surrounding tissue erosion, ${ }^{7}$ the present splint is smooth-walled and may offer a decreased erosion risk. 3D-printed PCL is less stiff than PTFE (elastic modulus 200 vs $350-700 \mathrm{MPa}$ ), a property that may also decrease
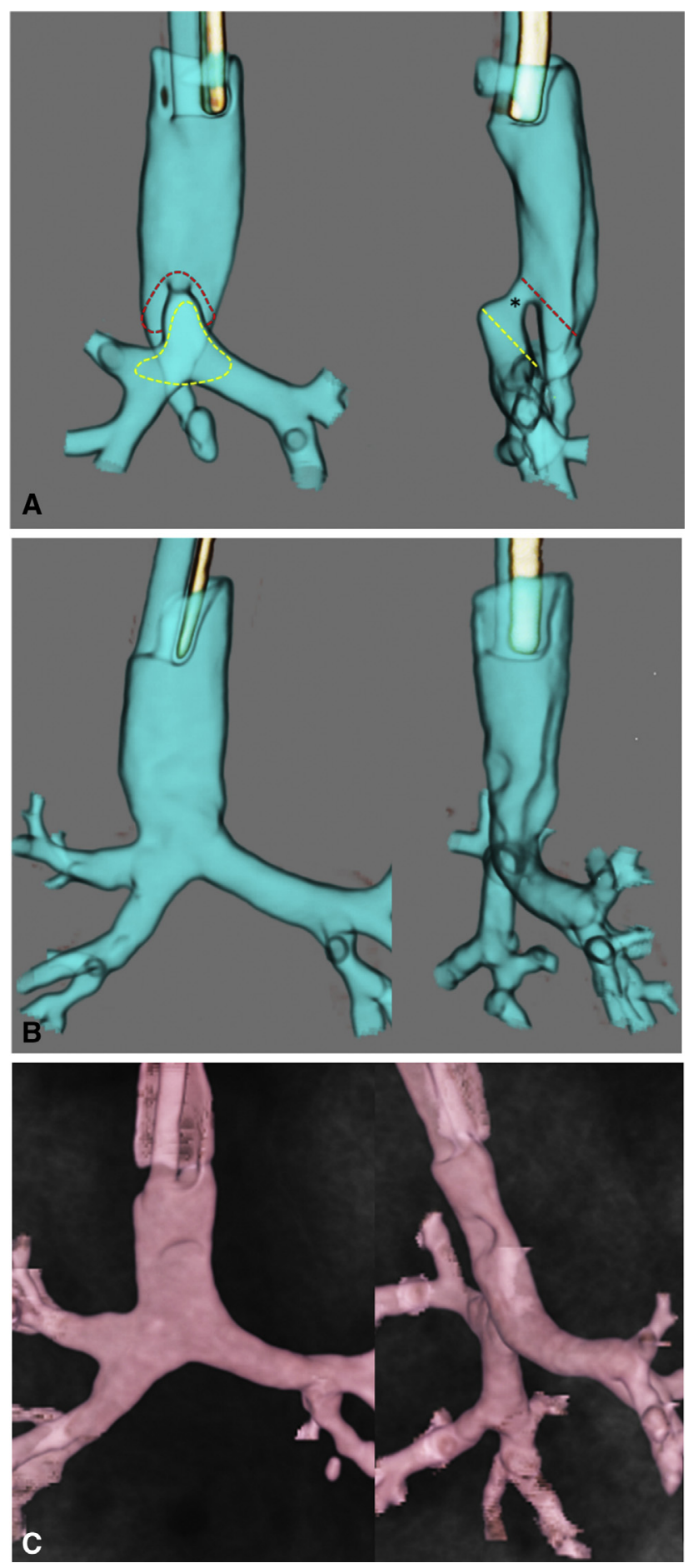

FIGURE 2. Computed tomography 3-dimensional reconstruction of airway before and after repair of tracheal agenesis (anterior and left lateral views). A, Airway before repair at age 2.6 months showing the $3.5-\mathrm{mm}$ diameter tracheoesophageal fistula (asterisk) and intended lines of division and anastomosis. B and C, Airway after repair at ages 8.6 and 23 months, respectively. 
erosion risk. Finally, bioresorbable materials may also be associated with decreased infection compared with permanent implants.

This novel management approach may contribute to better long-term airway outcomes for patients with TA. With coordinated multidisciplinary care and evolving technological advancements, extended survival may be possible for some patients with this rare and challenging disease.

The success of this case has significant ethical implications, as TA has traditionally been viewed as a fatal anomaly. Technological advancements such as 3D printing of bioresorbable implants may increase survival rate, albeit it is difficult to determine, given the rarity of the congenital deformity and its variations. Therefore, full disclosure of prognosis and potential outcomes to the family should not be overly optimistic despite previous successes.

The authors gratefully acknowledge Sarah Jo Crotts and Harsha Ramaraju, PhD, at 3DMedFab, Georgia Institute of Technology, for splint fabrication and assessment; The Global Center for Medical Innovation (GCMI) for quality control of splints; and
Catherine T. Haring, MD, Division of Otolaryngology, University of Michigan, for the drawing on the classification of tracheal agenesis.

\section{References}

1. Densmore JC, Oldham KT, Dominguez KM, Berdan ER, McCormick ME, Beste DJ, et al. Neonatal esophageal trachealization and esophagocarinoplasty in the treatment of flow-limited Floyd II tracheal agenesis. J Thorac Cardiovasc Surg. 2017;153:e121-5.

2. Smith MM, Huang A, Labbe M, Lubov J, Nguyen LHP. Clinical presentation and airway management of tracheal atresia: a systematic review. Int J Pediatr Otorhinolaryngol. 2017;101:57-64.

3. Tazuke Y, Okuyama H, Uehara S, Ueno T, Nara K, Yamanaka H, et al. Long-term outcomes of four patients with tracheal agenesis who underwent airway and esophageal reconstruction. J Pediatr Surg. 2015;50:2009-11.

4. Usui N, Kamiyama M, Tani G, Takama Y, Soh H, Uehara S, et al. Three-stage reconstruction of the airway and alimentary tract in a case of tracheal agenesis. Ann Thorac Surg. 2010;89:2019-22.

5. Les AS, Ohye RG, Filbrun AG, Ghadimi Mahani M, Flanagan CL, Daniels RC et al. 3D-printed, externally-implanted, bioresorbable airway splints for severe tracheobronchomalacia. Laryngosope. 2019;129:1763-71.

6. Baroncini-Cornea S, Fae M, Gargiulo G, Gentili A, Lima M, Pigna A, et al Tracheal agenesis: management of the first 10 months of life. Paediatr Anaesth. 2004:14:774-7.

7. Ando M, Nagase Y, Hasegawa H, Takahashi Y. External stenting: a reliable technique to relieve airway obstruction in small children. $J$ Thorac Cardiovasc Surg. 2017; $153: 1167-77$. 


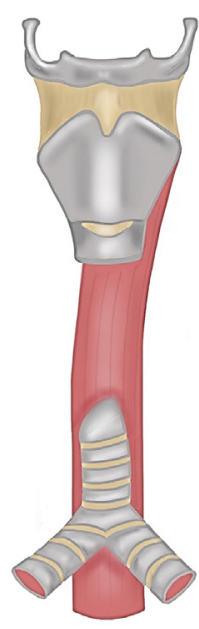

Type I

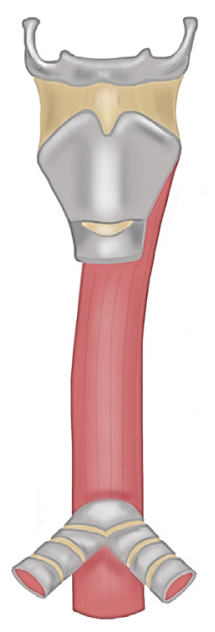

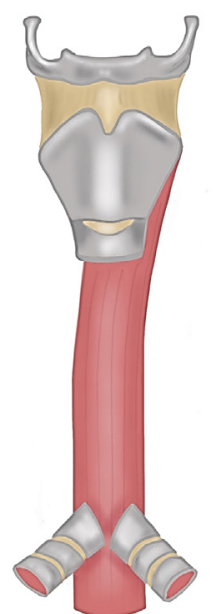

Type II

Type III

C. Haring

FIGURE E1. Floyd classification of tracheal agenesis.

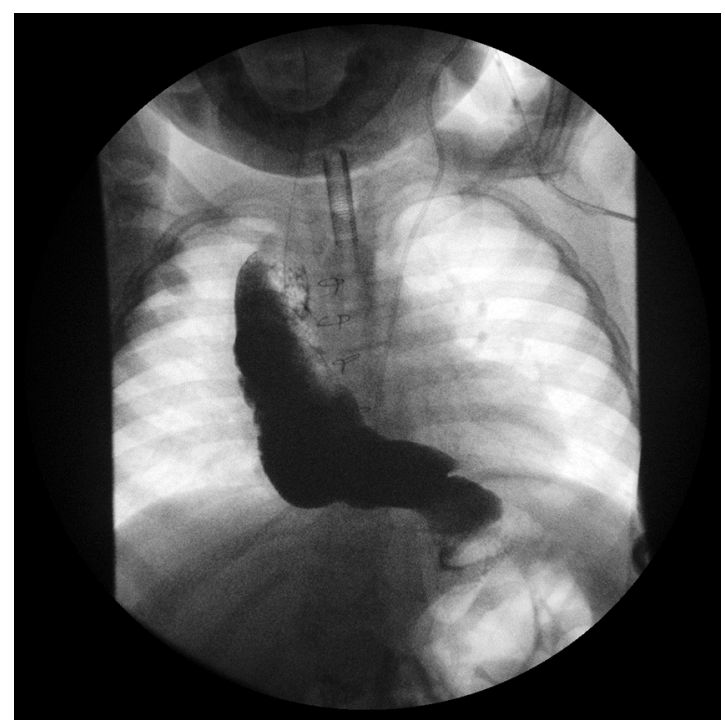

FIGURE E3. Upper gastrointestinal contrast study at age 2.7 years demonstrating establishment of gastrointestinal continuity between the cervical esophagus and the stomach with right colonic intrathoracic interposition graft.
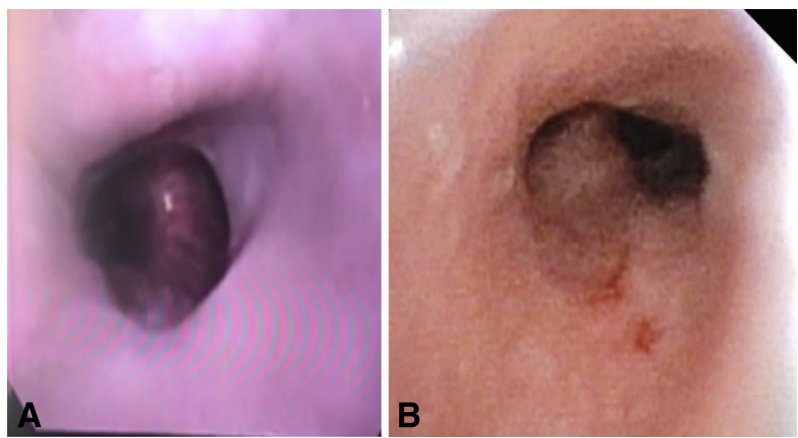

FIGURE E2. Bronchoscopy at 1 month (A) and 18 months (B) after esophagotracheoplasty with external splint placement showing the distal esophageal airway and the site of anastomosis above the carina. No airway collapse was observed with provocative suctioning. 